\title{
Nonlinear integrated Sachs-Wolfe effect
}

\author{
Asantha Cooray* \\ Department of Astronomy and Astrophysics, University of Chicago, Chicago, Illinois 60637 \\ and Sherman Fairchild Senior Research Fellow, California Institute of Technology, Pasadena, California 91125
}

(Received 7 August 2001; published 9 April 2002)

\begin{abstract}
We discuss the nonlinear extension to the integrated Sachs-Wolfe effect (ISW) resulting from the divergence of the large scale structure momentum density field. The nonlinear ISW effect leads to an increase in the total ISW contribution by roughly two orders of magnitude at $l \sim 1000$. This increase, however, is still below the cosmic variance limit of the primary anisotropies; at further small angular scales, secondary effects such as gravitational lensing and the kinetic Sunyaev-Zel'dovich effect dominate the nonlinear ISW power spectrum. We show that this second-order nonlinear ISW contribution is effectively the same as the contribution previously described as a lensing effect due to the transverse motion of gravitational lenses and well known as the Kaiser-Stebbins effect in the context of cosmic strings. Because of geometrical considerations, there is no significant three-point correlation function, or bispectrum, between the linear ISW effect and its nonlinear extension. The nonlinear ISW contribution can potentially be used as a probe of the transverse velocity of dark matter halos such as galaxy clusters. Because of the small contribution to temperature fluctuations, of the order of a few tenths of microkelvins, however, extracting useful measurements on velocities will be challenging.

DOI: 10.1103/PhysRevD.65.083518

PACS number(s): 98.80.Es, 95.85.Nv, 98.35.Ce, 98.70.Vc
\end{abstract}

\section{INTRODUCTION}

The importance of cosmic microwave background (CMB) temperature fluctuations as a probe of cosmology [1] is by now well known. The accuracy to which cosmological information can be extracted depends on how well we understand the individual processes that lead to anisotropies in the CMB temperature. Although effects during recombination are now well understood [2], contributions and modifications to CMB anisotropies due to large scale structure between the last scattering surface and today are not completely established. This is primarily due to the nonlinear evolution of the large scale structure at low redshifts, such that simple analytical calculations based on linear theory may no longer be applicable. In general, large scale structure affects the CMB through two processes: gravity and Compton scattering. The modifications due to gravity arise from frequency changes via gravitational red- and blueshifts, while during the reionized epoch photons can both generate and erase primary fluctuations through scattering via free electrons.

Here, we discuss an effect due to gravitational redshift commonly known in the literature as the integrated SachsWolfe (ISW [3]) effect at late times. ${ }^{1}$ The temperature fluctuations in the ISW effect result from the differential redshift effect from photons climbing in and out of time evolving potential perturbations from the last scattering surface to the present day. In currently popular cold dark matter cosmologies with a cosmological constant, significant contributions arise at redshifts less than 1 and on and above the scale of the horizon at the time of decay.

Here, in particular, we extend previous discussions on the

\footnotetext{
*Email address: asante@hyde.uchicago.edu

${ }^{1}$ To avoid confusion, we distinguish contributions to the ISW effect during the matter dominant era as the late ISW effect, although there is an additional contribution during radiation dominance.
}

ISW effect, usually due to linear fluctuations in the density field, to the nonlinear regime of clustering. The nonlinear contribution to the ISW effect is generally called the ReesSciama (RS) [4] effect and we discuss one aspect of the RS contribution involving the large scale structure density and velocity fields. Using the continuity equation, we show that a nonlinear contribution to the ISW effect comes from the divergence of the large scale structure momentum density field. We model the large scale structure momentum density field using the recently popular halo model where large scale structure density field fluctuations can be described through dark matter within halos and correlations between halos $[5,6]$. Our analytical calculations are consistent with those of [7] based on numerical simulations. Although we concentrate only on one aspect of the RS contribution, we note that there may be additional contributions: collapsing structures

In addition to a recalculation of the contribution, we also discuss this nonlinear extension to the ISW effect in the context of proposed contributions to the CMB temperature in the literature. We show that the nonlinear ISW contribution is essentially the same as the temperature anisotropy produced through transverse motions of foreground gravitational lenses, as was first discussed in Ref. [8], with further discussion and corrections in Ref. [9]. The effect is generally called the moving lens contribution [10,11] and its cosmic string analogue is the well known Kaiser-Stebbins effect [12]. Under this lensing description, the effect can be described as the gravitational lensing of the $\mathrm{CMB}$ dipole, formed by the transverse motion of a halo or a galaxy cluster (in the rest frame the halo). We show that there are no significant nonGaussian correlations associated with the nonlinear ISW effect and the bispectrum formed with linear effects is negligible. There is also no correlation between the kinetic Sunyaev-Zel'dovich (SZ) effect, due to the momentum density field along the line of sight, and the nonlinear ISW effect.

Note that we discuss only the contribution to the ISW 
effect due to large scale structure growth and evolution of structures by extending the well known result in the linear regime to the nonlinear regime. We note that there may be higher order contributions to the ISW effect, on both linear and nonlinear scales, as calculated in second-order perturbation theory for the geodesic equation [13]. Here, we ignore these corrections resulting from the second-order geodesic equation as the important ones were found to be small. Additionally, we note that there were previous attempts to calculate the nonlinear ISW contribution under various approximations to the large scale structure, including the "Swiss cheese" model of Ref. [14] and the void description of Ref. [15]. We note that such models may be unrealistic for the large scale structure and a more direct approach based on the formation and evolution of structures may be useful to consider instead.

The layout of the paper is as follows. In Sec. II, we outline the nonlinear contribution to the ISW effect after reviewing briefly background material relevant for current calculations in the context of adiabatic cold dark matter (CDM) models. In Sec. III, we discuss our results and consider the nonlinear contribution in the context of suggested secondary effects in the CMB. We also discuss the bispectrum formed by the combined linear ISW and its nonlinear extension. In the same section, we study the possibility of extraction of the transverse velocity of galaxy clusters using the nonlinear ISW effect.

\section{CALCULATIONAL METHOD}

We first review the properties of adiabatic CDM models relevant to the present calculations.

\section{A. Adiabatic CDM model}

The expansion rate for adiabatic CDM cosmological models with a cosmological constant is

$$
H^{2}=H_{0}^{2}\left[\Omega_{m}(1+z)^{3}+\Omega_{K}(1+z)^{2}+\Omega_{\Lambda}\right],
$$

where $H_{0}$ can be written as the inverse Hubble distance today, $H_{0}^{-1}=2997.9 h^{-1}$ Mpc. We follow the convention that, in units of the critical density $3 H_{0}^{2} / 8 \pi G$, the contribution of each component is denoted $\Omega_{i}, i=c$ for the CDM, $g$ for the baryons, and $\Lambda$ for the cosmological constant. We also define the auxiliary quantities $\Omega_{m}=\Omega_{c}+\Omega_{b}$ and $\Omega_{K}=1-\Sigma_{i} \Omega_{i}$, which represent the matter density and the contribution of spatial curvature to the expansion rate, respectively.

Convenient measures of distance and time include the conformal distance (or look-back time) from the observer at redshift $z=0$,

$$
r(z)=\int_{0}^{z} \frac{d z^{\prime}}{H\left(z^{\prime}\right)},
$$

and the analogous angular diameter distance

$$
d_{A}=H_{0}^{-1} \Omega_{K}^{-1 / 2} \sinh \left(H_{0} \Omega_{K}^{1 / 2} r\right) .
$$

Note that as $\Omega_{K} \rightarrow 0, d_{A} \rightarrow r$, and we define $r(z=\infty)=r_{0}$.
In linear theory, the density field may be scaled backward to higher redshift by the use of the growth function $G(z)$, where $\delta(k, r)=G(r) \delta(k, 0)[16]$,

$$
G(r) \propto \frac{H(r)}{H_{0}} \int_{z(r)}^{\infty} d z^{\prime}\left(1+z^{\prime}\right)\left(\frac{H_{0}}{H\left(z^{\prime}\right)}\right)^{3} .
$$

Note that in the matter dominated epoch $G \propto a=(1+z)^{-1}$.

Although we maintain generality in all derivations, we illustrate our results with the currently favored CDM with a cosmological constant $(\Lambda \mathrm{CDM})$. The parameters for this model are $\Omega_{c}=0.30, \Omega_{b}=0.05, \Omega_{\Lambda}=0.65, h=0.65, Y_{p}$ $=0.24, n=1$, and $X=1$, with a normalization such that mass fluctuation on the $8 \mathrm{~h} \mathrm{Mpc}^{-1}$ scale is $\sigma_{8}=0.9$, consistent with observations on the abundance of galaxy clusters [17] and Cosmic Background Explorer (COBE) normalization [18]. A reasonable value here is important since higher order correlations are nonlinearly dependent on the amplitude of the density field. To compute the linear power spectrum, we adopt the fitting formula for the transfer function given in [19].

\section{B. ISW effect}

The integrated Sachs-Wolfe effect [3] results from the late time decay of gravitational potential fluctuations. The resulting temperature fluctuations in the $\mathrm{CMB}$ can be written as

$$
T^{\mathrm{ISW}}(\hat{\mathbf{n}})=-2 \int_{0}^{r_{0}} d r \dot{\Phi}(r, \hat{\mathbf{n}} r),
$$

where the overdot represents the derivative with respect to conformal distance (or equivalently the look-back time). Writing multipole moments of the temperature fluctuation field $T(\hat{\mathbf{n}})$,

$$
a_{l m}=\int d \hat{\mathbf{n}} T(\hat{\mathbf{n}}) Y_{l}^{m} *(\hat{\mathbf{n}}),
$$

we can formulate the angular power spectrum as

$$
\left\langle a_{l_{1} m_{1}}^{*} a_{l_{2} m_{2}}\right\rangle=\delta_{l_{1} l_{2}}^{\mathrm{D}} \delta_{m_{1} m_{2}}^{\mathrm{D}} C_{l_{1}} .
$$

For the ISW effect, the multipole moments are

$$
a_{l m}^{\mathrm{ISW}}=i^{l} \int \frac{d^{3} \mathbf{k}}{2 \pi^{2}} \int d r \dot{\Phi}(\mathbf{k}) I_{l}(k) Y_{l}^{m}(\hat{\mathbf{k}}),
$$

with $I_{l}(k)=\int d r W^{\mathrm{ISW}}(k, r) j_{l}(k r)$, and the window function for the ISW effect $W^{\mathrm{ISW}}$ (see below). The angular power spectrum is then given by

$$
C_{l}^{\mathrm{ISW}}=\frac{2}{\pi} \int k^{2} d k P_{\dot{\Phi} \dot{\Phi}}(k)\left[I_{l}(k)\right]^{2},
$$

where the three-dimensional power spectrum of the timeevolving potential fluctuations is defined as

$$
\left\langle\dot{\Phi}\left(\mathbf{k}_{1}\right) \dot{\Phi}\left(\mathbf{k}_{2}\right)\right\rangle=(2 \pi)^{3} \delta_{D}\left(\mathbf{k}_{1}+\mathbf{k}_{2}\right) P_{\dot{\Phi} \dot{\Phi}}\left(k_{1}\right) .
$$


The above expression for the angular power spectrum can be evaluated efficiently under the Limber approximation [20] for sufficiently high $l$ values, usually in the order of a few tens, as

$$
C_{l}^{\mathrm{ISW}}=\int d r \frac{\left[W^{\mathrm{ISW}}\right]^{2}}{d_{A}^{2}} P_{\dot{\Phi} \dot{\Phi}}\left[k=\frac{l}{d_{A}}, r\right] .
$$

In order to calculate the power spectrum of the time derivative of potential fluctuations, we make use of the cosmological Poisson equation [21]. In Fourier space, we can relate the fluctuations in the potential to the density field as

$$
\Phi=\frac{3}{2} \frac{\Omega_{m}}{a}\left(\frac{H_{0}}{k}\right)^{2}\left(1+3 \frac{H_{0}^{2}}{k^{2}} \Omega_{K}\right)^{-2} \delta(k, r) .
$$

Thus, the derivative of the potential can be related to a derivative of the density field and the scale factor $a$. Considering a flat universe with $\Omega_{K}=0$, we can write the full expression for the power spectrum of time-evolving potential fluctuations, as is necessary for the ISW effect valid in all regimes of density fluctuations, as

$$
\begin{aligned}
P_{\dot{\Phi} \dot{\Phi}}(k, r)= & \frac{9}{4}\left(\frac{\Omega_{m}}{a}\right)^{2}\left(\frac{H_{0}}{k}\right)^{4}\left[\left(\frac{\dot{a}}{a}\right)^{2} P_{\delta \delta}(k, r)\right. \\
& \left.-2 \frac{\dot{a}}{a} P_{\delta \dot{\delta}}(k, r)+P_{\dot{\delta} \dot{\delta}}(k, r)\right]
\end{aligned}
$$

with $W^{\mathrm{ISW}}=-2$ in Eqs. (9) and (11).

To calculate the power spectrum involving the correlations between the time derivatives of density fluctuations $P_{\dot{\delta} \delta}$ and the cross-correlation term involving the density and time derivative of the density fields $P_{\delta \dot{\delta}}$, we make use of the continuity equation, which in position, or real, space can be written in the form

$$
\dot{\delta}(\mathbf{x}, r)=-\nabla \cdot[1+\delta(\mathbf{x}, r)] \mathbf{v}(\mathbf{x}, r) .
$$

In the linear regime of fluctuations, when $\delta(\mathbf{x}, r)$ $=G(r) \delta(\mathbf{x}, 0) \ll 1$, the time derivative is simply $\dot{\delta}^{\operatorname{lin}}(\mathbf{x}, r)$ $=-\nabla \cdot \mathbf{v}(\mathbf{x}, r)$ and we can obtain the well-known result for linear theory velocity fluctuations in Fourier space as

$$
\mathbf{v}=-i \dot{G} \delta(k, 0) \frac{\mathbf{k}}{k^{2}} .
$$

Thus, in linear theory, $P_{\dot{\delta} \dot{\delta}} \equiv k^{2} P_{v v}(k, r)=\dot{G}^{2} P_{\delta \delta}^{\operatorname{lin}}(k, 0)$ and $P_{\delta \dot{\delta}} \equiv k P_{\delta v}(k, r)=G \dot{G} P_{\delta \delta}^{\operatorname{lin}}(k, 0)$

These lead to the well-known results for the linear ISW effect with a power spectrum for $\dot{\Phi}$ as

$$
P_{\dot{\Phi} \dot{\Phi}}^{\operatorname{lin}}(k, r)=\frac{9}{4}\left(\frac{\Omega_{m}}{a}\right)^{2}\left(\frac{H_{0}}{k}\right)^{4}\left[-\frac{\dot{a}}{a} G(r)+\dot{G}\right]^{2} P_{\delta \delta}^{\operatorname{lin}}(k, 0) .
$$

The term within the square brackets is $\dot{F}^{2}$ where $F=G / a$ following the derivation for the linear ISW effect in [22].
Even though we have replaced the divergence of the velocity field with a time derivative of the growth function, it should be understood that the contributions to the ISW effect come from the divergence of the velocity field and not directly from the density field. Thus, to some extent, even the linear ISW effect reflects statistical properties of the large scale structure velocities.

In the mildly nonlinear to fully nonlinear regime of fluctuations, the approximation in Eq. (14), involving $\delta \ll 1$, is no longer valid and a full calculation of the time derivative of density perturbations is required. This can be achieved in the second-order perturbation theory, although such an approximation need not be fully applicable as the second-order perturbation theory fails to describe even the weakly nonlinear regime of fluctuations exactly. Motivated by applications of the halo approach to large scale structure $[5,6]$ and results from numerical simulations [23], we consider a description for the time derivative of density fluctuations and rewrite Eq. (14) as

$$
\dot{\delta}(\mathbf{x}, r)=-\boldsymbol{\nabla} \cdot \mathbf{v}(\mathbf{x}, r)-\boldsymbol{\nabla} \cdot \delta(\mathbf{x}, r) \mathbf{v}(\mathbf{x}, r),
$$

where we have separated the momentum term involving $p$ $=(1+\delta) v$ into a velocity contribution and a density velocity product. In Fourier space,

$$
\dot{\delta}(\mathbf{k})=i \mathbf{k} \cdot p(\mathbf{k})=i \mathbf{k} \cdot \mathbf{v}(\mathbf{k})+\int \frac{d^{3} \mathbf{k}^{\prime}}{(2 \pi)^{3}} \delta\left(\mathbf{k}-\mathbf{k}^{\prime}\right) i \mathbf{k} \cdot \mathbf{v}\left(\mathbf{k}^{\prime}\right)
$$

where we have dropped the time dependence for clarity. The first term involving the velocity field leads to the linear theory ISW effect, while the nonlinear aspects are captured in the term involving convolution of the $\delta v$ term (see also [7]). Following the approach motivated by $\mathrm{Hu}$ in Ref. [24], discussed by Cooray in Ref. [6], and investigated in detail through numerical simulations by Sheth et al. in Ref. [23], we can write the power spectrum of density derivatives, or equivalently the divergence of the momentum density field, as

$$
\begin{aligned}
P_{\delta \delta}(k) \equiv & k^{2} P_{p p}(k) \\
= & k^{2} P_{v v}^{\operatorname{lin}}(k)+k^{2} \int \frac{d^{3} \mathbf{k}^{\prime}}{(2 \pi)^{3}} \mu^{\prime 2} P_{\delta \delta}\left(\left|\mathbf{k}-\mathbf{k}^{\prime}\right|\right) P_{v v}\left(k^{\prime}\right) \\
& +k^{2} \int \frac{d^{3} \mathbf{k}^{\prime}}{(2 \pi)^{3}} \frac{\left(k-k^{\prime} \mu^{\prime}\right) \mu^{\prime}}{\left|\mathbf{k}-\mathbf{k}^{\prime}\right|} P_{\delta v} \\
& \times\left(\left|\mathbf{k}-\mathbf{k}^{\prime}\right|\right) P_{\delta v}\left(k^{\prime}\right)+k^{2} \int \frac{d^{3} \mathbf{k}^{\prime}}{(2 \pi)^{3}} \int \frac{d^{3} \mathbf{k}^{\prime \prime}}{(2 \pi)^{3}} \mu^{\prime} \mu^{\prime \prime} \\
& \times T_{\delta \delta v v}\left(\mathbf{k}-\mathbf{k}^{\prime},-\mathbf{k}-\mathbf{k}^{\prime \prime}, \mathbf{k}^{\prime}, \mathbf{k}^{\prime \prime}\right) .
\end{aligned}
$$

In the nonlinear regime, $\mathbf{k}-\mathbf{k}^{\prime} \sim \mathbf{k}$ and $\mathbf{k}-\mathbf{k}^{\prime \prime} \sim \mathbf{k}$ and we can simplify by integrating over angles to obtain 


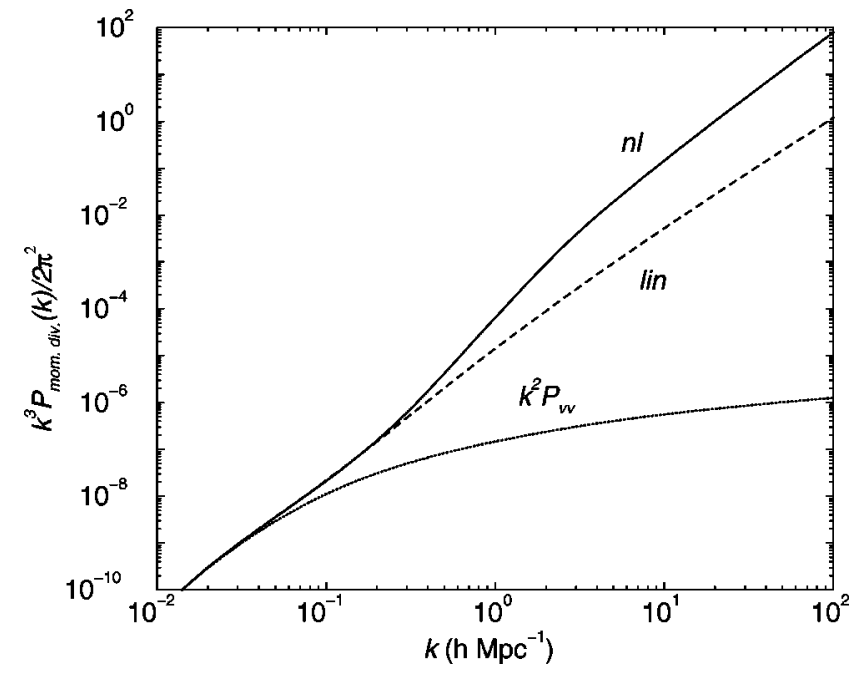

FIG. 1. The power spectrum of the divergence of the momentum density field $P_{\dot{\delta} \dot{\delta}}(k)$. Here, we show the contribution due to the divergence of the velocity field and the extension to the nonlinear regime using Eq. (20).

$$
P_{\dot{\delta} \dot{\delta}}=k^{2} P_{v v}^{\operatorname{lin}}(k)+\frac{1}{3} k^{2} P_{\delta \delta}(k) \int \frac{k^{\prime 2} d k^{\prime}}{2 \pi^{2}} P_{v v}\left(k^{\prime}\right)
$$

Note that in the deeply nonlinear regime contributions from the trispectrum formed by the velocity-density correlations drop out; this is due to the fact that, under the halo approach, the nonlinear trispectrum resulting from the single halo term is independent of the configuration and thus of $\mu^{\prime}$ and $\mu^{\prime \prime}$ (see the discussions in [6] and [25]). Also, the term involving the product of cross-power spectra between the density and velocity fields does not contribute in the nonlinear regime; at small scales, the density fluctuations are independent of the large scale velocity field. In Fig. 1, we show the power spectrum of momentum divergence, which in linear theory is simply described by the divergence of the velocity field, with the extension to the nonlinear regime of fluctuations following Eq. (20).

Our description of the nonlinear momentum power spectrum is similar to the derivation of the nonlinear kinetic SZ effect by $\mathrm{Hu}$ [24] and Cooray [6] (see also [23]) which involves the momentum field along the line of sight. In Eq. (20), the integral over the velocity power spectrum is simply the rms of the velocity fluctuations

$$
v_{r m s}^{2}=\int d k \frac{P_{\delta \delta}^{\operatorname{lin}}(k)}{2 \pi^{2}} .
$$

Thus, the nonlinear power spectrum of the momentum field divergence, as relevant for the ISW effect, involves one of three components of the velocity field with $1 / 3 v_{\text {rms }}^{2}$; note that the expression for the fully nonlinear contribution to the momentum field along the line of sight is similar to the above and also involves one component of the velocity field as discussed in [24] and [6]. The resulting expression for the nonlinear momentum field is fully consistent with simulations [23].

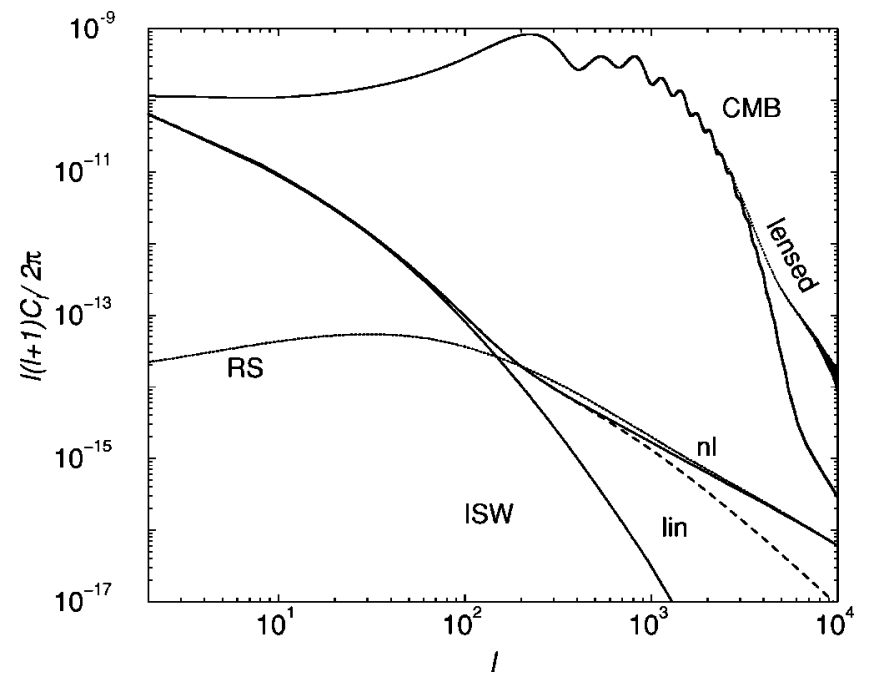

FIG. 2. The angular power spectrum of the full ISW effect, including nonlinear contribution. The contribution called ReesSciama (RS) shows the nonlinear extension, although for the total contribution, the cross term between the momentum field and the density field leads to a slight suppression between $l$ of 100 and 1000. The curve labeled "nl" is the full nonlinear contribution while the curve labeled "lin" is the contribution resulting from the momentum field under second-order perturbation theory.

In addition to the power spectrum of density derivatives, in Eq. (13) we also require the cross-power spectrum between the density derivatives and density field itself, $P_{\delta \dot{\delta}}$. In [23], using the halo approach as a description of the momentum density field observed in simulations, it was found that the cross correlation between the density field and the momentum field can be well described as

$$
P_{p \delta}(k)=\sqrt{P_{p p}(k) P_{\delta \delta}(k)} .
$$

This is equivalent to the statement that the density and momentum density fields are perfectly correlated with a crosscorrelation coefficient of 1 ; this relation is exact at mildly linear scales while at deeply nonlinear scales this perfect cross correlation requires a mass independent specific velocity for individual halos [23]. Using this observation, we make the assumption that $P_{\delta \dot{\delta}} \sim \sqrt{P_{\delta \delta} P_{\dot{\delta} \dot{\delta}}}$, which is generally reproduced in the halo model description of the cross correlation between the density field and density field derivatives. This cross term leads to a $10 \%$ reduction of power at multipoles between 100 and 1000, when compared to the total when linear and nonlinear contributions are simply added.

\section{DISCUSSION}

In Fig. 2, we show the angular power spectrum of the ISW effect with its nonlinear extension (which we have labeled RS for Rees-Sciama effect). The curve labeled "ISW" is the simple linear theory calculation with a power spectrum for potential derivatives given in Eq. (16). The curves labeled "lin" and "nl" show the full nonlinear calculation following the description given in Eq. (13) and using the linear theory or full nonlinear power spectrum, in Eq. (20), for the 


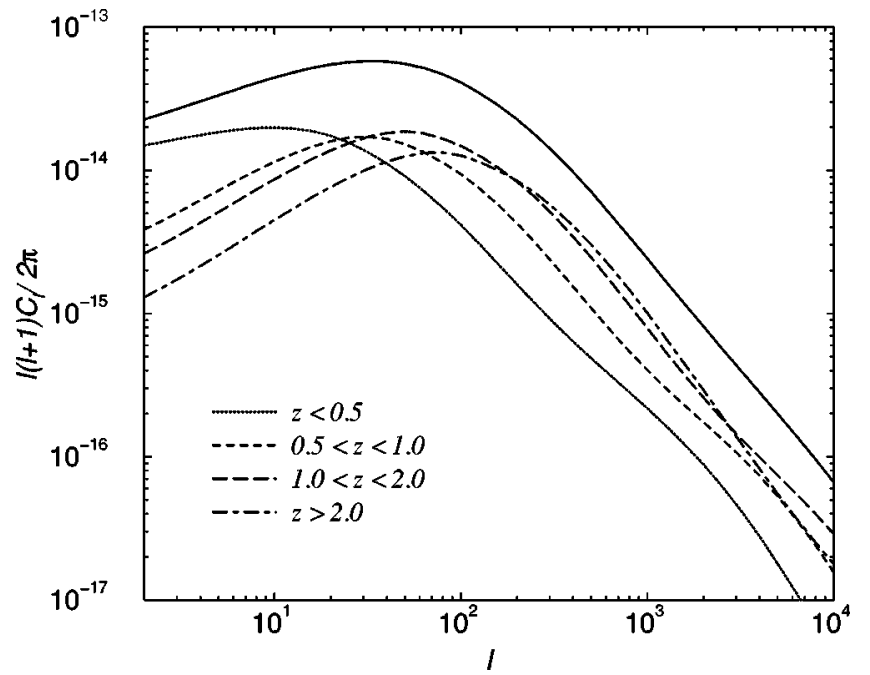

FIG. 3. The redshift dependence of the nonlinear ISW contribution. Here, we break down the contributions at $z$ less than 0.5 (dotted line), between 0.5 and 1.0 (dashed line), between 1.0 and 2.0 (long dashed line), and for redshifts greater than 2 (dot-dashed line). As shown, most of the contributions arise at a redshift of $\sim 1$, leading to the peak in power at $l \sim 100$ and a sharp decrease thereafter.

density field, respectively. For the nonlinear density field power spectrum, we make use of the halo approach for large scale structure clustering $[5,6]$ and calculate the power spectrum through a distribution of dark matter halos. We use linear theory to describe the velocity field in both linear and nonlinear cases; since the velocity field only contributes as an overall normalization, through $v_{\text {rms }}$, its nonlinear effects, usually at high $k$ values, are not important due to the shape of the velocity power spectrum and the behavior of the integral in Eq. (21).

As shown in Fig. 2, the overall correction due to the nonlinear ISW effect leads to roughly a two order of magnitude increase in power at $l \sim 1000$. The difference between the linear and nonlinear theory density field power spectra in Eq. (20) leads only to at most an order of magnitude change in power. Note that the curve labeled "lin" agrees with previous second-order perturbation theory calculations of the Rees-Sciama effect [7], while the curve labeled "nl" is also consistent with previous estimates based on results from numerical simulations.

There is an additional feature that should be observed in Fig. 2. At $l \sim 100$ to 1000 , there is roughly a $10 \%$ decrease in total power. This is due to the $P_{\delta \delta}$ term in Eq. (13). If one simply adds the linear ISW and nonlinear RS contributions, this cross-correlation term is not present. This dip, due to a cancellation, is present when comparing results based on perturbation theory for RS and numerical simulations for the full nonlinear ISW effect (see [7]); the cross term provides a natural explanation for the slight decrease in power.

In Fig. 3, we breakdown the contribution to the nonlinear effect, without the linear ISW or cross-term contributions, into a function of steps in redshift. As shown, one essentially finds equal contributions over a wide range in redshift with most of the contributions coming from a redshift $\sim 1$ to mul-
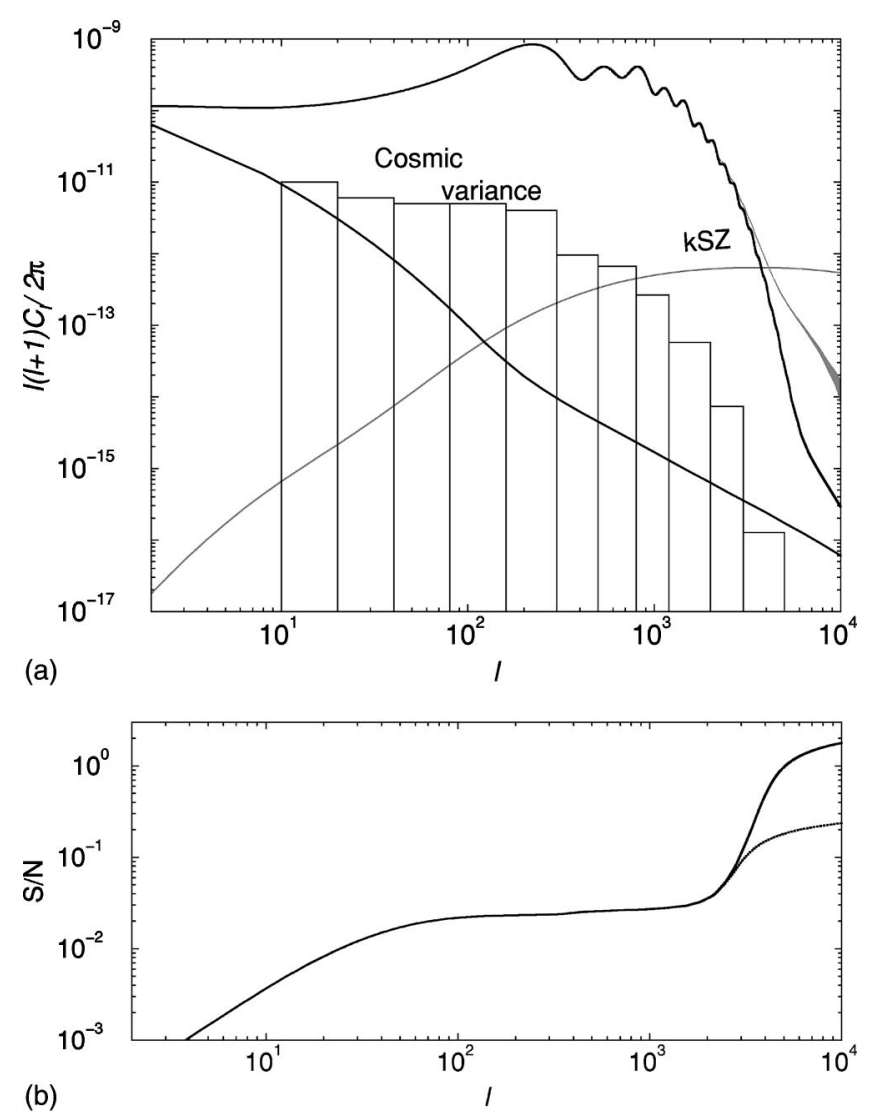

FIG. 4. (Top) The total ISW contribution compared with cosmic variance due to primary anisotropies. As shown, the contributions are far below the cosmic variance and the nonlinearities are not likely to impact the upcoming measurements of cosmological parameters from $\mathrm{CMB}$ data. At small angular scales, where the nonlinear contributions are well above the sample variance, other secondary effects, such as the kinetic SZ effect, dominate the temperature fluctuations. (Bottom) Cumulative signal-to-noise ratio for the detection of the nonlinear ISW power spectrum, in the presence of the SZ thermal effect (dotted line) and not (solid line), and for an all sky experiment with no detector noise contribution.

tipoles of a few hundred where the power peaks.

Even though there is roughly two orders of magnitude increase in power at multipoles around 1000, in Fig. 4, we show that this increase is still below the cosmic variance associated with the primary contribution given by

$$
\Delta C_{l}=\sqrt{\frac{2 f_{\mathrm{sky}}^{-1}}{2 l \Delta_{l}+\Delta_{l}^{2}}} C_{l}^{\mathrm{CMB}},
$$

where $\Delta_{l}$ is the bin size in multipole space and $f_{\text {sky }}$ is the fraction of sky covered. The signal-to-noise ratio for the detection of the power spectrum is

$$
\left(\frac{S}{N}\right)^{2}=\frac{f_{\text {sky }}}{2} \sum_{l}(2 l+1)\left(\frac{C_{l}}{C_{l}^{n}}\right)^{2}
$$


where $C_{l}^{n}$ is the power spectrum of noise with $C_{l}^{n}=C_{l}^{\mathrm{CMB}}$ $+C_{l}^{\mathrm{s}}+C_{l}^{\text {det }} ; C_{l}^{\mathrm{s}}$ is any contribution from secondary effects and $C_{l}^{\text {det }}$ is any detector noise contribution. As shown in Fig. 4(b), the cumulative signal-to-noise ratio is less than 1 even for a full sky experiment with $f_{\text {sky }}=1$ and no detector noise. Here, we include contributions from the thermal and kinetic Sunyaev-Zel'dovich effects (KSZ) [26,6] and the lensing effect on CMB [27] as secondary contributions to the noise power spectrum. The top line with cumulative signal-tonoise ratio slightly above 1 is when the thermal SZ effect is removed from the noise contribution.

Given the significant sample variance and the fact that the nonlinear ISW effect has no special property, such as a different frequency spectrum from the thermal CMB from the case of the thermal SZ effect [26,28], it is unlikely that the nonlinear ISW effect power spectrum can be extracted from CMB data easily. Going to much smaller angular scales, or large multipoles, leads to a reduction in the cosmic variance, although the increase in power associated with other small angular scale secondary effects such as the thermal SZ effect or the kinetic SZ effect can complicate any detection of the nonlinear ISW effect. Later, we will address the possibility whether, instead of statistical properties such as the power spectrum or the bispectrum (see below), we can extract the associated signal from individual objects such as massive galaxy clusters.

\section{A. ISW bispectrum}

Following our earlier discussion for the angular power spectrum, we can also consider the bispectrum (see [29]), or the Fourier analogue of the three-point correlation function:

$$
\begin{aligned}
B(\hat{\mathbf{n}}, \hat{\mathbf{m}}, \hat{\mathbf{l}}) & \equiv\langle T(\hat{\mathbf{n}}) T(\hat{\mathbf{m}}) T(\hat{\mathbf{l}})\rangle \\
& \equiv \sum\left\langle a_{l_{1} m_{1}} a_{l_{2} m_{2}} a_{l_{3} m_{3}}\right\rangle Y_{l_{1}}^{m_{1}}(\hat{\mathbf{n}}) Y_{l_{2}}^{m_{2}}(\hat{\mathbf{m}}) Y_{l_{3}}^{m_{3}}(\hat{\mathbf{l}}),
\end{aligned}
$$

where the sum is over $\left(l_{1}, m_{1}\right),\left(l_{2}, m_{2}\right),\left(l_{3}, m_{3}\right)$. Statistical isotropy again allows us to express the correlation in terms of an $m$-independent function,

$$
\left\langle a_{l_{1} m_{1}} a_{l_{2} m_{2}} a_{l_{3} m_{3}}\right\rangle=\left(\begin{array}{ccc}
l_{1} & l_{2} & l_{3} \\
m_{1} & m_{2} & m_{3}
\end{array}\right) B_{l_{1} l_{2} l_{3}} .
$$

Here the quantity in parentheses is the Wigner $3 j$ symbol. The orthonormality relation for the Wigner $3 j$ symbol implies

$$
B_{l_{1} l_{2} l_{3}}=\sum_{m_{1} m_{2} m_{3}}\left(\begin{array}{ccc}
l_{1} & l_{2} & l_{3} \\
m_{1} & m_{2} & m_{3}
\end{array}\right)\left\langle a_{l_{1} m_{1}} a_{l_{2} m_{2}} a_{l_{3} m_{3}}\right\rangle .
$$

For the coupling between ISW effects, using the multipolar moments written in Eq. (8), we can write the bispectrum as

$$
\begin{aligned}
B_{l_{1} l_{2} l_{3}}= & \sqrt{\frac{\left(2 l_{1}+1\right)\left(2 l_{2}+1\right)\left(2 l_{3}+1\right)}{4 \pi}} \\
& \times\left(\begin{array}{ccc}
l_{1} & l_{2} & l_{3} \\
0 & 0 & 0
\end{array}\right) b_{l_{1}, l_{2}, l_{3}},
\end{aligned}
$$

with

$$
\begin{aligned}
b_{l_{1}, l_{2}, l_{3}}= & \frac{2^{3}}{\pi^{3}} \int k_{1}^{2} d k_{1} \int k_{2}^{2} d k_{2} \int k_{3}^{2} d k_{3} B_{\dot{\Phi} \dot{\Phi} \dot{\Phi}} \\
& \times\left(k_{1}, k_{2}, k_{3}\right) I_{l_{1}}\left(k_{1}\right) I_{l_{2}}\left(k_{2}\right) I_{l_{3}}\left(k_{3}\right) \\
& \times \int x^{2} d x j_{l_{1}}\left(k_{1} x\right) j_{l_{2}}\left(k_{2} x\right) j_{l_{3}}\left(k_{3} x\right) .
\end{aligned}
$$

As before, the Limber approximation [20] allows one to simplify the integrals for speedy calculation:

$$
\begin{aligned}
& b_{l_{1}, l_{2}, l_{3}}=\int d r \frac{W^{\mathrm{ISW}}\left(k_{1}, r\right) W^{\mathrm{ISW}}\left(k_{2}, r\right) W^{\mathrm{ISW}}\left(k_{3}, r\right)}{d_{A}^{4}} \\
& \times\left. B_{\dot{\Phi} \dot{\Phi} \dot{\Phi}}\left(k_{1}, k_{2}, k_{3}\right)\right|_{k_{1}=l_{1} / d_{A}, k_{2}=l_{2} / d_{A}, k_{3}=l_{3} / d_{A}} .
\end{aligned}
$$

Similar to the power spectrum in Eq. (10), the threedimensional bispectrum of the derivatives of potential fluctuations is defined as

$$
\begin{aligned}
& \left\langle\dot{\Phi}\left(k_{1}\right) \dot{\Phi}\left(k_{2}\right) \dot{\Phi}^{\mathrm{nl}}\left(k_{3}\right)\right\rangle \\
& \quad=(2 \pi)^{3} \delta_{D}\left(\mathbf{k}_{1}+\mathbf{k}_{2}+\mathbf{k}_{3}\right) B_{\dot{\Phi} \dot{\Phi} \dot{\Phi}}\left(k_{1}, k_{2}, k_{3}\right) .
\end{aligned}
$$

As an approximation, this three-dimensional bispectrum can be calculated in second-order perturbation theory (e.g., [30]). To obtain an exact result valid in both the weakly nonlinear and nonlinear regimes, we consider the coupling between two linear ISW effects and the nonlinear extension involving the $\boldsymbol{\nabla} \cdot \delta \mathbf{v}$ term. This leads to the following bispectrum:

$$
\begin{aligned}
B_{\dot{\Phi} \dot{\Phi} \dot{\Phi}}\left(k_{1}, k_{2}, k_{3}\right)= & -\frac{27}{8}\left(\frac{\Omega_{m}}{a}\right)^{3} \frac{H_{0}^{6}}{k_{1}^{2} k_{2}^{2} k_{3}^{2}}\left[-\frac{\dot{a}}{a} G+\dot{G}\right]^{2} \\
& \times G \dot{G}\left[P_{\delta \delta}\left(k_{1}, 0\right) P_{\delta v}\left(k_{2}, 0\right)\right. \\
& \left.\times \frac{\mathbf{k}_{3} \cdot \mathbf{k}_{2}}{k_{2}}+\text { perm. }\right]
\end{aligned}
$$

where the permutations are with respect to the ordering of $\mathbf{k}_{1}, \mathbf{k}_{2}$, and $\mathbf{k}_{3}$ leading to a total of six terms. In Eq. (30), $W^{\mathrm{ISW}}=-2$ as defined earlier. Because of the dependence on an angle, say $\mathbf{k}_{3} \cdot \mathbf{k}_{2}$ in $B_{\dot{\Phi} \dot{\Phi} \dot{\Phi}}\left(k_{1}, k_{2}, k_{3}\right)$, there are significant cancellations and the final projected angular bispectrum of the ISW effect is smaller than a simple order of magnitude estimate involving the cube of the temperature fluctuation 


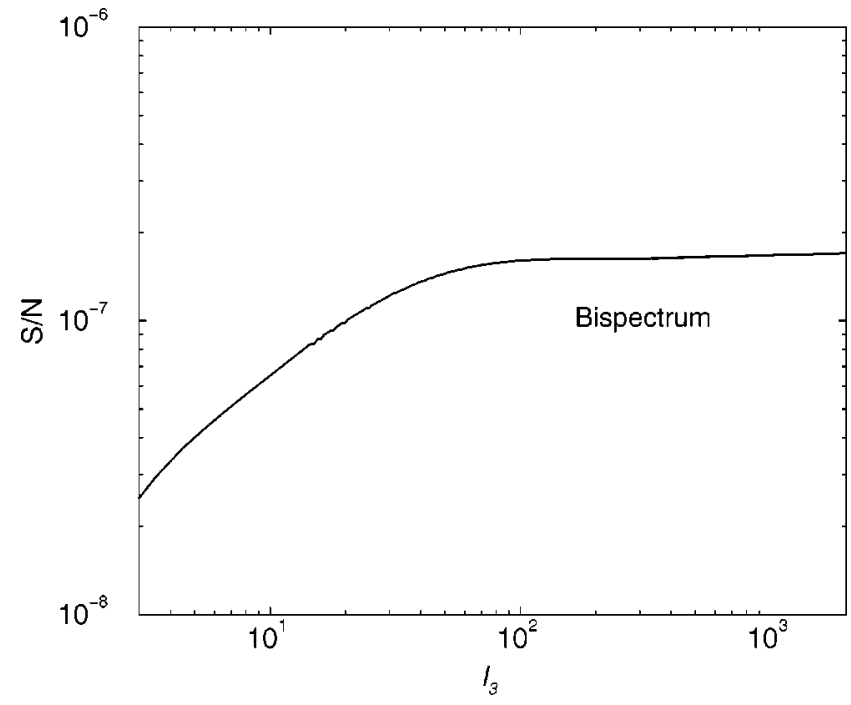

FIG. 5. The cumulative signal-to-noise ratio for the detection of the nonlinear ISW effect bispectrum as a function of the multipole $l_{3}$.

amplitude. In Fig. 5, we show the cumulative signal-to-noise ratio for the ISW bispectrum, with

$$
\left(\frac{S}{N}\right)^{2}=f_{\text {sky }} \sum_{l_{1}, l_{2}, l_{3}} \frac{B_{l_{1} l_{2} l_{3}}^{2}}{6 C_{l_{1}}^{n} C_{l_{2}}^{n} C_{l_{3}}^{n}},
$$

where again the noise power spectrum is $C_{l}^{n}$ (see Ref. [22] for details). In Fig. 5, we take $C_{l}^{n}=C_{l}^{\mathrm{CMB}}$ to consider sample variance from primary anisotropies alone. The cumulative signal-to-noise ratio is on the order of a few times $10^{-7}$, suggesting that the bispectrum is unlikely to be detected; similar values are also found for other three-point statistics such as the skewness or the third moment. Thus, consistent with the second-order perturbation theory result [30], there is no significant non-Gaussian signal at the three-point level formed by the correlation between linear ISW effects and the nonlinear ISW effect in the nonlinear regime of fluctuations. Also note that there is no bispectrum of the form $\left\langle\dot{\Phi}\left(k_{1}\right) \dot{\Phi}^{\mathrm{nl}}\left(k_{2}\right) \dot{\Phi}^{\mathrm{nl}}\left(k_{3}\right)\right\rangle$ since such a term leads to an odd number of velocity or density fluctuation terms.

\section{B. Cross correlations}

An additional way to extract or detect the presence of this signal is through cross correlations with another source of anisotropy or a tracer of large scale structure. Since the momentum density field is involved in the nonlinear ISW effect, one can expect the presence of a correlation between another tracer of the momentum density field. It is well known that the kinetic SZ effect traces the line of sight large scale structure momentum density field such that the temperature fluctuations can be written as a modulation of the velocity field by baryon fluctuations,

$$
T^{\mathrm{KSZ}}(\hat{\mathbf{n}})=\int d r g(r) \hat{\mathbf{n}} \cdot \mathbf{v}(r, \hat{\mathbf{n}} r) \delta_{b}(r, \hat{\mathbf{n}} r),
$$

where $\delta_{b}$ is the fluctuation in the baryon field and $g(r)$ is a weight function for Compton scattering with $g \equiv \dot{\tau} e^{-\tau}$ $=X H_{0} 0.0691\left(1-Y_{p}\right) \Omega_{g} h(1+z)^{2} e^{-\tau}$ where $\tau(r)=\int_{0}^{r} d r \dot{\tau}$ is the optical depth out to $r[31,22,6]$.

The correlation between the nonlinear ISW effect and the kinetic SZ effect is then

$$
\left\langle a_{l_{1} m_{1}}^{*, \mathrm{ISW}} a_{l_{2} m_{2}}^{\mathrm{KSZ}}\right\rangle \propto \int \frac{d^{3} \mathbf{k}^{\prime}}{(2 \pi)^{3}} \mu^{\prime} \sqrt{1-\mu^{\prime 2}} P_{\delta b}(\mathbf{k}) P_{v v}\left(\mathbf{k}^{\prime}\right),
$$

and is equal to zero through the angular terms involving the integral over $\mathbf{k} \cdot \mathbf{k}^{\prime}=k k^{\prime} \mu^{\prime}$; the cross correlation involves a cosine term from the divergence of the momentum associated with the nonlinear ISW effect and a sine term from the line of sight momentum associated with the kinetic SZ effect. The geometrical cancellation is merely a statement that, although locally the line of sight velocity field may be correlated with its transverse component, on average over the whole sky there is no such correlation. This cancellation can be avoided by several techniques. In [6], we discussed a similar situation involving the cross correlation between the thermal SZ effect and the kinetic SZ effect and suggested the use of a quadratic correlation involving the square of the density field. This is also equivalent to the use of absolute values of the temperature fluctuations.

\section{Relation to other effects}

We now suggest that the nonlinear extension to the ISW effect is essentially the same contribution described by [8] using the transverse motion of lensing objects; in the context of cosmic strings, this contribution is well known as the Kaiser-Stebbins effect [12]. Writing $T(\hat{\mathbf{n}})=\int d r \Delta T(r \hat{\mathbf{n}}, r)$, we consider the ISW effect such that $\Delta T(r \hat{\mathbf{n}}, r)=-2 \Phi(r)$. Following our earlier discussion related to the nonlinear contribution, we can relate the potential fluctuations to the density fluctuations using the Poisson equation. The contributions now follow as $\Delta T \propto[-\dot{a} / a \delta+\dot{\delta}]$. The first term containing the time derivative of the scale factor was recently reintroduced by [32] as a time-delay effect. As discussed in [33], the time-delay contribution to the CMB is second order as it involves a product of the spatial gradient of the CMB at the last scattering surface and the cumulative time-delay contribution and is not simply described by a first-order contribution. Additionally, as discussed for the linear ISW effect, considering only the $\dot{a}$ term leads to an overestimate of the fluctuation as there is a cancellation from a first-order term involving the $\dot{\delta}$ term.

As before, using the complete continuity equation, we can relate the time derivative of the density fluctuations $\dot{\delta}$ to the divergence of the velocity field and the divergence of the product of overdensity and velocity $\Delta T \propto[\dot{a} / a \delta+\nabla \cdot(1$ $+\delta) \mathbf{v}]$ Here, we consider the nonlinear contribution resulting from the $\Delta T^{\mathrm{nl}} \propto \boldsymbol{\nabla} \cdot \delta \mathbf{v}$ term and reintroduce the density field in terms of potentials using the Poisson equation, 


$$
\Delta T^{\mathrm{nl}}=-2 \boldsymbol{\nabla} \cdot(\Phi \mathbf{v}) \approx-\mathbf{v}_{\perp} \cdot\left(2 \nabla_{r} \Phi\right)=-v \sin \alpha \delta_{\mathrm{len}} \cos \phi .
$$

The simplifications assume that potential fluctuations are embedded in a velocity field with much larger coherence scale so that gradients in the velocity field do not contribute to temperature anisotropies. Furthermore, we have introduced the lensing deflection angle $\delta_{\text {len }}=2 \nabla_{r} \Phi$ where the gradient is now an angular gradient on the sky and there is no contribution to temperature anisotropy from the gradient of the potential along the line of sight. This forces the contributing component of the velocity field to be the one on the sky and not along the line of sight. Here, $\alpha$ is the angle between the line of sight and the velocity field and $\phi$ is the position angle from the observer. As is now clear, this latter description of the nonlinear ISW effect is what has been provided elsewhere in the context of moving gravitational lenses [10,11].

The correspondence between the two effects can also be noted using the description that is well utilized to calculate the gravitational lensing effect on the CMB [27,22,34]. Writing

$$
T(\hat{\mathbf{n}})=T(\hat{\mathbf{n}}+\Delta \hat{\mathbf{n}})=T^{\mathrm{P}}(\hat{\mathbf{n}})+\nabla_{r} T^{\mathrm{P}}(\hat{\mathbf{n}}) \cdot \Delta \hat{\mathbf{n}}
$$

where angular deflections due to gravitational lensing are given by $\Delta \hat{\mathbf{n}}=2 \nabla_{r} \phi$, where the projected lensing potential is $\phi(\hat{\mathbf{n}})=D_{l s} / D_{s} \Phi$. Here, $T^{\mathrm{P}}(\hat{\mathbf{n}})$ is the primary CMB contribution while $\nabla_{r} T^{\mathrm{P}}(\hat{\mathbf{n}})$ is the angular gradient on the sky of these temperature fluctuations. Essentially, gravitational lensing angular deflections remap the distribution of temperature fluctuations, and this remapping is captured by the dependence on distances, where the distance from lens to source (CMB last scattering surface) is $D_{l s}$ and the distance from observer to source is $D_{s}$. Also, as written, the gravitational lensing effect on the CMB is second order due to the dependence on the angular gradient of the CMB on the sky, $\nabla_{r} T$; this is consistent with the fact that lensing does not change the surface brightness and results only in a modification of the temperature fluctuation distribution.

The second-order effect related to the nonlinear ISW effect is due to the lensing of the dipole created by the motion of halos such that $\nabla_{r} T=v \sin \alpha$. To be consistent with the description given using the time derivative of the density fluctuations, we require $D_{l s}=D_{s}$, such that there is no dependence on the ratio of distance factors, usually encountered in lensing studies. This is equivalent to the case that $D_{l} \ll D_{s}$ such that $D_{l s} \sim D_{s}$, which is not necessarily true even for clusters at $z \sim 0.5$. In either case, the nonlinear ISW effect cannot be considered as a remapping of the temperature fluctuations similar to the conventional gravitational lensing, as there is no real source, a temperature gradient, in the problem; the temperature gradient essentially exists from the coordinate transformation from a moving lens to a stationary lens. ${ }^{2}$ An inconsistency with the lensing mapping de-

\footnotetext{
${ }^{2}$ We note that this issue has led to some confusion in the literature when calculating the so-called moving lens effect, with some including the ratio of $D_{l s} / D_{s}[10]$ while others, correctly, do not [11].
}

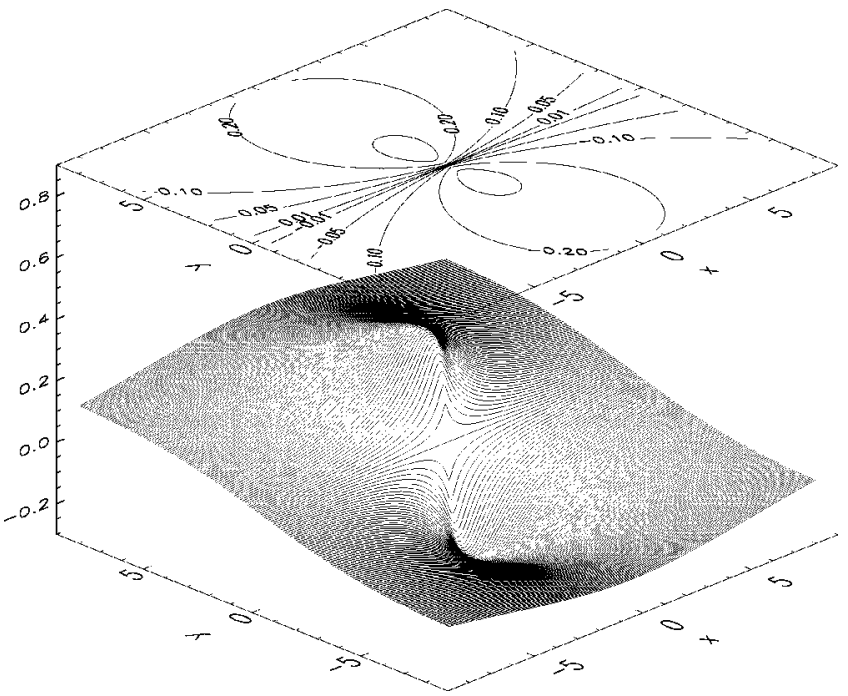

FIG. 6. The contribution due to the nonlinear ISW effect for a cluster of mass $5 \times 10^{14} M_{\odot}$ with a transverse velocity of $100 \mathrm{~km} \mathrm{~s}^{-1}$ across the line of sight. The temperature fluctuations produce a distinct dipolar pattern on the sky and are of the order of a few tenths of microkelvins. Here, the $x$ and $y$ coordinates are in terms of the scale radius of the cluster, based on a NFW profile.

scription arises, unfortunately, when attempting to describe the contribution from the divergence of the momentum density field as a gravitational lensing effect.

\section{Toward transverse velocities}

As discussed before, the detection of the power spectrum of temperature anisotropies due to the nonlinear ISW effect is heavily affected by the dominant cosmic variance of the CMB primary anisotropies. From geometrical considerations, there is also no significant non-Gaussian contribution or cross correlation with other effects. Thus, instead of statistical properties such as the power spectrum, one can try to extract the associated signal in individual objects such as massive galaxy clusters.

In Fig. 6, we show the distinct signature formed by the nonlinear ISW effect for a cluster of mass $5 \times 10^{14} M_{\odot}$ with a transverse velocity of $100 \mathrm{~km} \mathrm{~s}^{-1}$ across the line of sight. We assume a Navarro-Frenk-White (NFW) [35] profile for the dark matter distribution of the cluster and take a description for the concentration-mass relation following [36]. In terms of the density distribution of the cluster, the deflection angle, at an impact distance of $\eta$ from the cluster center, is given by

$$
\delta_{\text {len }}(\eta)=\frac{8 \pi G}{c^{2} \eta} \int_{0}^{\eta} r_{\perp} d r_{\perp} \sum\left(r_{\perp}\right)
$$

where the surface mass density is

$$
\sum\left(r_{\perp}\right)=\int_{-r_{v}}^{+r_{v}} d r_{\|} \rho\left(r_{\perp}, r_{\|}\right) .
$$


In the above, $r_{\perp}$ is the distance across the line of sight, $r_{\|}$is the distance along the line of sight, and $r_{v}$ is the virial radius of the cluster, which we take to be at an overdensity of 200 following the NFW [35] description. The above equation for the deflection angle also assumes a circularly symmetric dark matter distribution within the halo.

As shown in Fig. 6, the contribution to temperature fluctuations is at most $0.3 \mu \mathrm{K}$. In order to detect this small signal, one has to be able to extract it from other contributions to the $\mathrm{CMB}$ due to galaxy clusters. The most significant contributions from clusters arise from the SZ thermal effect [26] due to inverse Compton scattering of photons via hot electrons. Here, temperature changes of the order of $1 \mathrm{mK}$ are produced and these are now routinely observed toward massive clusters [37]. The SZ thermal effect, however, has a distinct frequency dependence, and in multifrequency CMB data the effect can be separated out from thermal CMB and other fluctuations [28]. The next significant contribution comes from the kinetic SZ effect due to the line of sight motion of the scatterers in clusters [26]. In Fig. 7, we show the kinetic SZ effect for the same cluster as in Fig. 6. Here, we have assumed that the electron distribution in clusters is described by hydrostatic equilibrium [6] and have taken a line of sight velocity of $100 \mathrm{~km} \mathrm{~s}^{-1}$. The contribution due to the SZ kinetic effect, and also the SZ thermal effect, is highly peaked toward the center of the cluster and can be as high as a few tens of microkelvins.

The next important contribution is due to gravitational lensing of the large scale CMB gradient, whose rms is of order $13 \mu \mathrm{K} \operatorname{arcmin}^{-1}$. With a deflection angle of order $\sim 0.5$ arcmin, the contribution due to lensing is in the range of a few microkelvins. As shown in Fig. 7, the lensing effect has the same profile shape as the contribution resulting from the transverse velocity; the two profiles need not lie in the same direction since the large scale CMB gradient and the transverse velocities can be aligned differently. When the thermal SZ effect is separated, in temperature fluctuations, a galaxy cluster exhibits a slightly offset dipolar pattern with a significant temperature increment or decrement toward the center, resulting from the direction of the line of sight velocity associated with the kinetic SZ effect. Detecting such a profile will certainly remain a challenging goal for future cluster observations [38].

Eventually, if the transverse velocity contribution can be detected, its amplitude and the direction of the dipole pattern will provide significant information on large scale structure velocities not generally available from other observations. We can ask how well one can detect a typical cluster through this effect. First, to make a reasonable signal-to-noise detection, it is clear that one must extract effects such as the kinetic SZ and CMB lensing. This requires detailed knowledge of the baryon and dark matter distributions within clusters. Assuming such information is available, we can obtain an estimate for the signal-to-noise ratio by noting that the observed signal can be written as $[38,39]$

$$
\frac{\Delta T}{T}(\theta)=s(\theta)+n(\theta),
$$
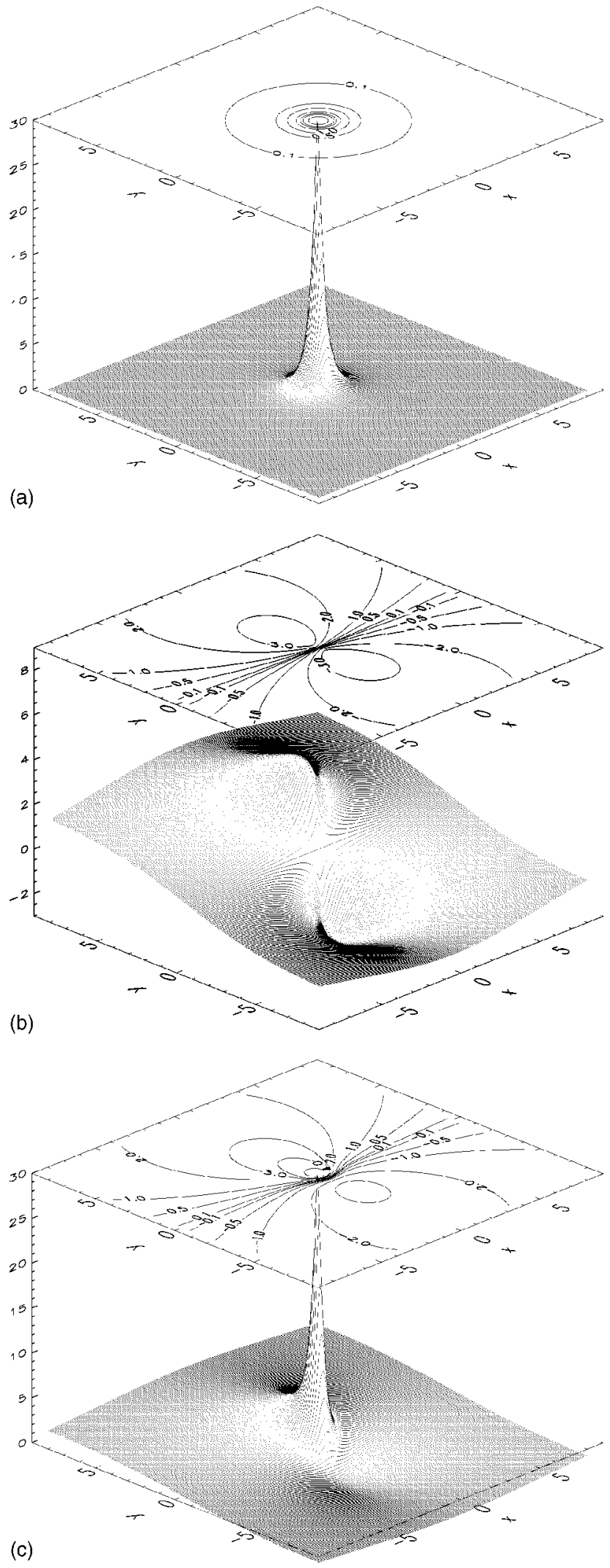

FIG. 7. Temperature fluctuations due to clusters. Top: Kinetic SZ effect; middle: lensing of CMB primary temperature fluctuations; and bottom: total contribution from kinetic SZ, lensing, and transverse velocities. We use the same cluster as shown in Fig. 6. 


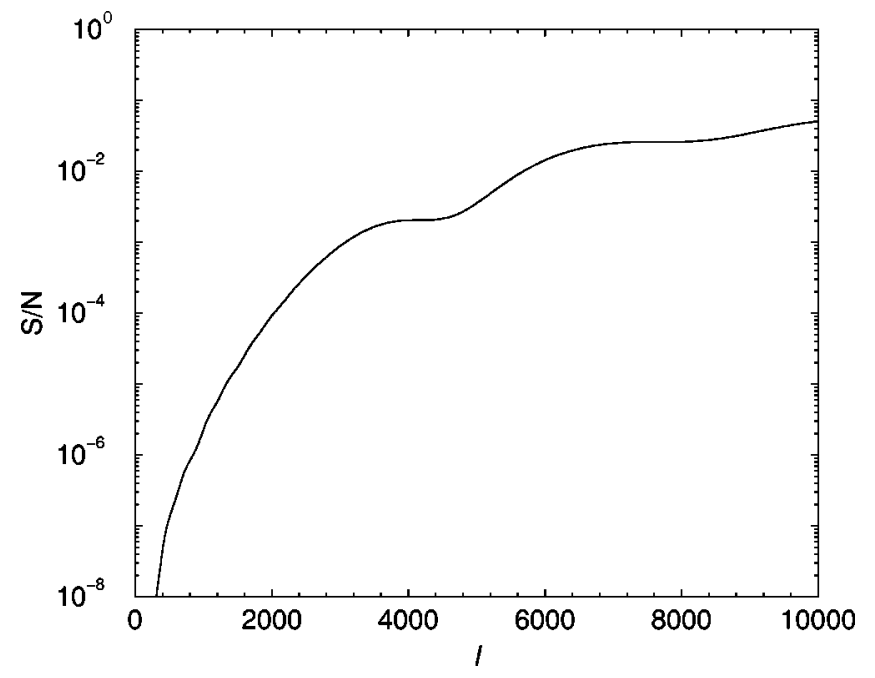

FIG. 8. The signal-to-noise ratio for the detection of the nonlinear ISW effect. The galaxy cluster considered here is the one in Fig. 6.

where $s(\theta)$ is the profile of the signal and $n(\theta)$ is the profile of the noise distribution. In order to remove the excess noise associated with large scale temperature fluctuations from primary anisotropies or other secondary effects, we can construct an appropriately normalized filter which provides an optimal detection of the signal in the presence of such noise. This filter can be written as

$$
\Psi(\mathbf{l})=\frac{s(\mathbf{l})}{C_{l}^{n}}\left[\int \frac{d^{2} \mathbf{l}}{(2 \pi)^{2}} \frac{|s(\mathbf{l})|^{2}}{C_{l}^{n}}\right]^{-1}
$$

where $C_{l}^{n}$ is the power spectrum of the noise. The signal-tonoise ratio for the detection of the profile, for an axisymmetric distribution for $s(\theta)$, is

$$
\left(\frac{S}{N}\right)^{2}=\int \frac{l d l}{2 \pi} \frac{s(l)^{2}}{C_{l}^{n}} .
$$

In Fig. 8, we show the cumulative signal-to-noise ratio as a function of $l$. Here, we assume a noise power spectrum given by the sum of the intrinsic CMB and secondary effects, where secondary effects include all thermal contributions: CMB lensing, kinetic SZ, and a model for the inhomogeneous reionization. At small $l$ 's, corresponding to the outer extent of the cluster, fluctuations in the intrinsic CMB temperature confuse the detection of the signal, while at large $l$ 's, corresponding to the inner extent of the cluster, fluctuations from the local universe complicate it. Even if detailed properties of clusters are known so that intrinsic CMB lensing and the kinetic SZ effect can be perfectly separated from the transverse effect, it is unlikely that we will know all sources of temperature fluctuation along the line of sight toward a given cluster, leading to confusion in the detection. For typical transverse velocities of order a few $100 \mathrm{~km} \mathrm{~s}^{-1}$, we find signal-to-noise values of the order of 0.1 suggesting that detection of this signal for individual clusters will remain challenging even for an experiment with no instrumen- tal noise contributions; alternatively, one can put an upper limit on the transverse velocity contribution at the level of a few thousand $\mathrm{km} \mathrm{s}^{-1}$; this upper limit is considerably larger than what one expects under currently popular $\Lambda \mathrm{CDM}$ cosmological models.

The signal-to-noise ratio for detection may be improved if cross-correlation techniques can be considered, e.g., if one has some knowledge of the intrinsic and secondary fluctuations toward the observed cluster and of the direction of the large scale bulk flows from other methods, even if the amplitude of that bulk flow is not known. Separately, since the coherence scales of bulk flows are much larger than an individual cluster, it may be possible to extract the transverse effect by averaging the signal over a number of clusters. Using numerically simulated cluster images, and realistic sources of noise and confusion, we plan to study how well such an extraction can be performed in a future paper.

\section{SUMMARY}

We have discussed the nonlinear extension to the integrated Sachs-Wolfe effect resulting from the divergence of the large scale structure momentum density field. This nonlinear ISW effect, calculated under the recently popular halo approach to nonlinear large scale structure clustering, leads to an increase in the total ISW contribution by roughly two orders of magnitude at $l \sim 1000$. This increase, however, is still below the cosmic variance limit of the primary anisotropies; at smaller angular scales, secondary effects such as gravitational lensing and the kinetic Sunyaev-Zel'dovich effect dominate the nonlinear ISW power spectrum.

Further, we have shown that this second-order nonlinear ISW contribution is effectively the same as the contribution previously described as a lensing effect due to the transverse motion of gravitational lenses and well known as the KaiserStebbins effect related to cosmic strings. From geometrical considerations, there is no significant three-point correlation function, or bispectrum, between the linear ISW effect and its nonlinear counterpart. The correlation between the nonlinear ISW effect and the kinetic SZ effect is again zero due to the geometry associated with the line of sight and divergence of the momentum density field. The nonlinear ISW contribution can potentially be used as a probe of the transverse velocity of dark matter halos such as galaxy clusters; however, because of the small contribution to temperature fluctuations of the order of a few tenths of microkelvins, extracting useful measurements on velocities will be challenging.

\section{ACKNOWLEDGMENTS}

We are grateful to Wayne $\mathrm{Hu}$ for useful discussions. We thank Ravi Sheth and his collaborators for providing us with an early draft of their paper on a halo model description of the velocity and momentum density fields and for useful conversations. We also thank Albert Stebbins for his encouragement of this work. A.C. was supported by a NASA grant NAG5-10840 at Chicago and the Fairchild Foundation and DOE at Caltech. 
[1] L. Knox, Phys. Rev. D 52, 4307 (1995); G. Jungman, M. Kamionkowski, A. Kosowsky, and D. N. Spergel, ibid. 54, 1332 (1996); J. R. Bond, G. Efstathiou, and M. Tegmark, Mon. Not. R. Astron. Soc. 291, L33 (1997); M. Zaldarriaga, D. N. Spergel, and U. Seljak, Astrophys. J. 488, 1 (1997); D. J. Eisenstein, W. Hu, and M. Tegmark, ibid. 518, 2 (1999).

[2] W. Hu, N. Sugiyama, and J. Silk, Nature (London) 386, 37 (1997).

[3] R. K. Sachs and A. M. Wolfe, Astrophys. J. 147, 73 (1967).

[4] M. J. Rees and D. N. Sciama, Nature (London) 519, 611 (1968).

[5] R. J. Scherrer and E. Bertschinger, Astrophys. J. 381, 349 (1991); R. K. Sheth and B. Jain, Mon. Not. R. Astron. Soc. 285, 231 (1997); U. Seljak, ibid. 318, 203 (2000); C.-P. Ma and J. N. Fry, Astrophys. J. 543, 502 (2000); A. Cooray and W. Hu, ibid. 548, 7 (2001); R. Scoccimarro, R. Sheth, L. Hui, and B. Jain, ibid. 546, 20 (2001).

[6] A. Cooray, Ph.D. thesis, University of Chicago, 2001 (available from the U. of Chicago Crear Science Library or from the author); Phys. Rev. D 64, 063514 (2001).

[7] U. Seljak, Astrophys. J. 460, 549 (1996); see also R. Tuluie and P. Laguna, Astrophys. J. Lett. 445, L73 (1995); R. Tuluie, P. Laguna, and P. Anninos, Astrophys. J. 463, 15 (1996).

[8] M. Birkinshaw and S. F. Gull, Nature (London) 302, 24 (1983).

[9] L. I. Gurvits and I. G. Mitrofanov, Nature (London) 324, 27 (1986); M. Birkinshaw, in Moving Gravitational Lenses, edited by J. Moran, J. Hewitt, and K. Y. Lo (Springer-Verlag, Berlin, 1989), p. 59.

[10] N. Aghanim, S. Prunet, O. Forni, and F. R. Bouchet, Astron. Astrophys. 334, 409 (1998).

[11] S. M. Molnar and M. Birkinshaw, Astrophys. J. 537, 542 (2000).

[12] N. Kaiser and A. Stebbins, Nature (London) 310, 2 (1984); A. Stebbins, Astrophys. J. 327, 584 (1988).

[13] T. Pyne and S. M. Carroll, Phys. Rev. D 53, 2920 (1996); S. Mollerach and S. Matarrese, ibid. 56, 4494 (1997).

[14] L. Nottale, Mon. Not. R. Astron. Soc. 206, 713 (1984); E. Martińez-González and J. L. Sanz, ibid. 247, 473 (1990).

[15] K. L. Thompson and E. T. Vishniac, Astrophys. J. 313, 517 (1987); C. C. Dyer and P. S. S. Ip, Mon. Not. R. Astron. Soc. 235, 895 (1988).

[16] P. J. E. Peebles, The Large-Scale Structure of the Universe (Princeton University Press, Princeton, NJ, 1980).
[17] P. T. P. Viana and A. R. Liddle, Mon. Not. R. Astron. Soc. 303, 535 (1999).

[18] E. F. Bunn and M. White, Astrophys. J. 480, 6 (1997).

[19] D. J. Eisenstein and W. Hu, Astrophys. J. 511, 5 (1999).

[20] D. Limber, Astrophys. J. 119, 655 (1954).

[21] J. M. Bardeen, Phys. Rev. D 22, 1882 (1980).

[22] A. Cooray and W. Hu, Astrophys. J. 534, 533 (2000).

[23] R. K. Sheth, A. Diaferio, and I. Zehavi (unpublished); see also C.-P. Ma and J. N. Fry, astro-ph/0106342.

[24] W. Hu, Astrophys. J. 529, 12 (1999).

[25] A. Cooray and W. Hu, Astrophys. J. 554, 56 (2001).

[26] R. A. Sunyaev and Ya. B. Zel'dovich, Mon. Not. R. Astron. Soc. 190, 413 (1980).

[27] U. Seljak, Astrophys. J. 463, 1 (1996); M. Zaldarriaga and U. Seljak, Phys. Rev. D 58, 023003 (1998); W. Hu, ibid. 62, 043007 (2000); W. Hu and A. Cooray, ibid. 63, 023504 (2001).

[28] A. Cooray, W. Hu, and M. Tegmark, Astrophys. J. 540, 1 (2000).

[29] E. Komatsu and D. N. Spergel, Phys. Rev. D 63, 063002 (2001); L. Wang and M. Kamionkowski, ibid. 61, 063504 (1999); A. Gangui and J. Martin, Mon. Not. R. Astron. Soc. 313, 323 (2000); X. Luo and D. N. Schramm, Phys. Rev. Lett. 71, 1124 (1993); X. Luo, Astrophys. J. Lett. 427, L71 (1994); T. Falk, R. Rangaranjan, and M. Frednicki, ibid. 403, L1 (1993).

[30] D. N. Spergel and D. M. Goldberg, Phys. Rev. D 59, 103001 (1999).

[31] J. P. Ostriker and E. T. Vishniac, Nature (London) 322, 804 (1986); E. T. Vishniac, Astrophys. J. 322, 597 (1987).

[32] C. Da-Ming, X.-P. Wu, and D.-R. Jiang, Astrophys. J. 544, 1 (2000).

[33] W. Hu and A. Cooray, Phys. Rev. D 63, 023504 (2001).

[34] D. M. Goldberg and D. N. Spergel, Phys. Rev. D 59, 103002 (1999).

[35] J. Navarro, C. Frenk, and S. D. M. White, Astrophys. J. 462, 563 (1996).

[36] J. S. Bullock, T. S. Kolatt, and Y. Sigad, Mon. Not. R. Astron. Soc. (to be published), astro-ph/9908159; Y. P. Jing, Astrophys. J. 535, 30 (2000).

[37] J. E. Carlstrom, M. Joy, and L. Grego, Astrophys. J. Lett. 456, L75 (1996); M. Jones et al., Nature (London) 365, 320 (1993).

[38] U. Seljak and M. Zaldarriaga, astro-ph/9907254.

[39] M. G. Haehnelt and M. Tegmark, Mon. Not. R. Astron. Soc. 279, 545 (1996). 